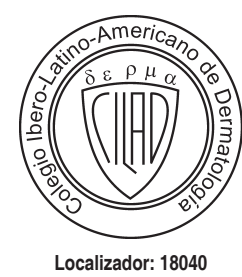

doi: $10.35366 / 91762$

\title{
Torus Palatino
}

\author{
Torus Palatinus
}

Valeria Elizabeth Rossello, ${ }^{*}$ María Noelia Andrade, ${ }^{\ddagger}$ Virginia Ruth López Gamboa, ${ }^{\S}$ María Julia Blanzari, ${ }^{\S}$ María Susana Gómez Zanni, ${ }^{\S}$ Mariana Beatriz del Valle Papa

\section{REsUMEN}

El torus palatino se encuentra dentro de las hiperplasias óseas benignas del macizo facial, conjuntamente con el torus mandibular y las exostosis óseas. Son generalmente de etiología desconocida, asintomáticas y no requieren tratamiento por su benignidad. Pueden ser un diagnóstico accidental y obligan a descartar patologías neoplásicas. Presentamos el caso de una paciente con diagnóstico de torus palatino multilobulado.

\section{ABSTRACT}

Torus palatinus is a type of benign hyperplasia of the facial skeleton, together with torus mandibularis and bony exostoses. These are mostly of unknown etiology and asymptomatic; in addition, they do not require treatment due to their benignity. Their diagnosis may be incidental and any neoplastic pathologies must be ruled out. We present a case of a patient diagnosed with multilobular torus palatinus.

\section{INTRODUCCIÓN}

$\mathrm{E}^{\prime}$ torus palatino es una hiperplasia de masa ósea ${ }^{1-3}$ que se presenta en el paladar duro, ${ }^{1-6}$ representando una variación anatómica de carácter benigno más que una condición patológica. ${ }^{1,6}$

\section{PRESENTACIÓN DEL CASO}

* Residente de tercer año de Dermatología.

Residente segundo año de Dermatología.

$\S$ Especialista en

Dermatología, médica de planta.

" Jefa del Servicio de

Dermatología.

Clínica Universitaria Reina Fabiola, Córdoba, Argentina

Conflicto de intereses: Ninguno.

Recibido:

03/Junio/2018.

Aceptado:

10/Septiembre/2018

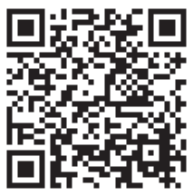

Mujer de 57 años de edad sin antecedentes personales patológicos. Consultó al Servicio de Dermatología de nuestra institución para control de nevos y al examen físico de mucosa oral palatina se evidenció una lesión tumoral, exofítica, lobulada de $1.5 \times 1 \mathrm{~cm}$ de diámetro localizada a nivel del rafe medio palatino, asintomática, de consistencia pétrea, recubierta de mucosa de aspecto sano, de aproximadamente seis meses de evolución (Figura 1). Por su apariencia clínica, se plantearon los diagnósticos diferenciales de torus palatino vs neoplasias óseas o de partes blandas, por lo que se solicitó tomografía de macizo facial (Figura 2), que confirmó el diagnóstico de torus palatino multilobulado. En vista de que la paciente se encontraba asintomática y por la característica benigna de la enfermedad, se decidió derivación al Servicio de Odontología para control evolutivo, quienes indicaron placa siliconada de descanso nocturno.

\section{DISCUSIÓN}

La prevalencia del torus palatino es de $10 \%$ en la población general ${ }^{1,3,4,7}$ y varía en relación a la edad, género y etnia, ${ }^{1-3}$ siendo más baja en América de Sur.? Se observó mayor incidencia en mujeres, ${ }^{1-3,5,6}$ con predominio de pacientes postmenopáusicas ${ }^{1,6}$ y con mayor frecuencia entre los 39-59 años de edad. ${ }^{3}$ Si bien su etiología no está completamente establecida, existen teorías ligadas a factores genéticos y ambientales. ${ }^{1,5}$ Varias investigaciones sugieren la transmisión de herencia autosómico-dominante, ${ }^{1-3,5,7}$ mientras que otros sólo lo asocian al estrés oclusal severo o a la relación de ambos factores. También se describen hábitos alimenticios involucrados con el crecimiento del tejido óseo, ${ }^{1,2}$ con una mayor prevalencia en aquéllos que consumen ácidos grasos poliinsaturados omega-3 y vitamina D. . $^{2,7}$

Clínicamente son tumoraciones de consistencia pétrea, recubiertos de una mucosa fina de color normal, ${ }^{3}$ que por lo general son descubiertos en el examen odontológico clínico de rutina debido a que la mayoría de los casos son asintomáticos..$^{1,4,5}$ Estos hallazgos son compatibles con la clínica de nuestra paciente. Microscópicamente, están constituidos por láminas de hueso compacto y centro de hueso esponjoso. ${ }^{2,5}$ Su crecimiento es lento y según su 
morfología pueden ser planos, lobulados, multilobulados, nodulares o fusiformes, siendo esta última la forma más común. ${ }^{4}$ Algunos autores lo clasificaron según su ubicación (Tabla 1). ${ }^{7}$ La paciente que aquí se describe presentaba un torus palatino multilobulado tipo IV, por su ubicación en zona posterior del maxilar.

El diagnóstico del TP generalmente se realiza teniendo en cuenta sus características clínicas ${ }^{1,5}$ y en la mayoría de los casos no requiere de métodos diagnósticos adicionales. ${ }^{5}$ Muchas veces se observa en una imagen radiográfica

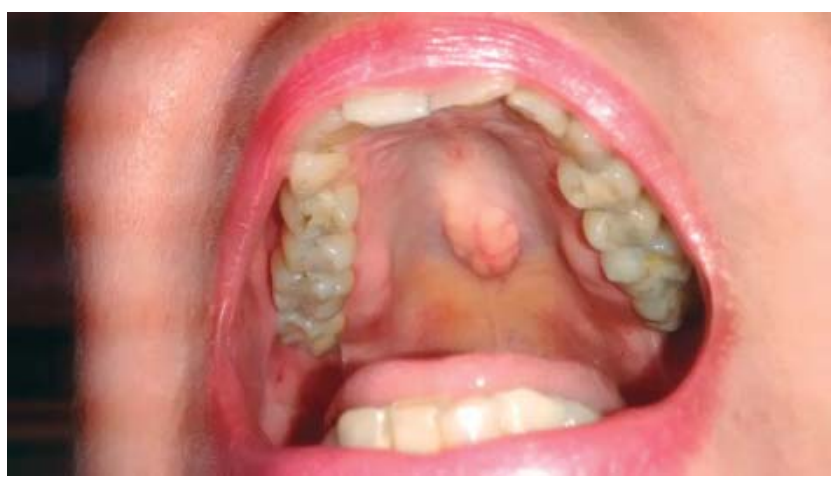

Figura 1: Torus palatino multilobulado recubierto de mucosa normal en región media palatina. como un defecto radiopaco. ${ }^{8,9}$ Sin embargo por sus diagnósticos diferenciales, para permitir una mejor evaluación y por su relación con estructuras anatómicas circundantes, puede solicitarse una tomografía axial computada (TAC). ${ }^{1,5}$ En la TAC se identifican protuberancias óseas con densidad similar a la del hueso compacto ${ }^{5}$ tal como las imágenes observadas en nuestra paciente. La biopsia raramente es necesaria debido a las características clínicas que generalmente son patognomónicas. ${ }^{1}$

Dentro de los diagnósticos diferenciales debemos considerar: osteoma característico del síndrome de Gardner, odontoma, ${ }^{5}$ fibroma osificante o cementoma maduro, el hematoma subperióstico osificado, el callo óseo no resuelto, el osteosarcoma precoz y el condrosarcoma precoz. ${ }^{8,10}$ Con escasa frecuencia suelen asociarse a enfermedades como la adenomatosis colónica, síndrome de Maffucci, la enfermedad de Ollier y los osteocondromas múltiples..$^{8,9}$

Tabla 1. Clasificación de TP según su ubicación.

\begin{tabular}{ll} 
Tipo & Ubicación \\
\hline I & Foramen incisivo a espina nasal posterior \\
II & Foramen incisivo a la sutura palatina transversal \\
III & Zona posterior del maxilar a la parte posterior de espina nasal \\
IV & Zona posterior del maxilar \\
\hline
\end{tabular}

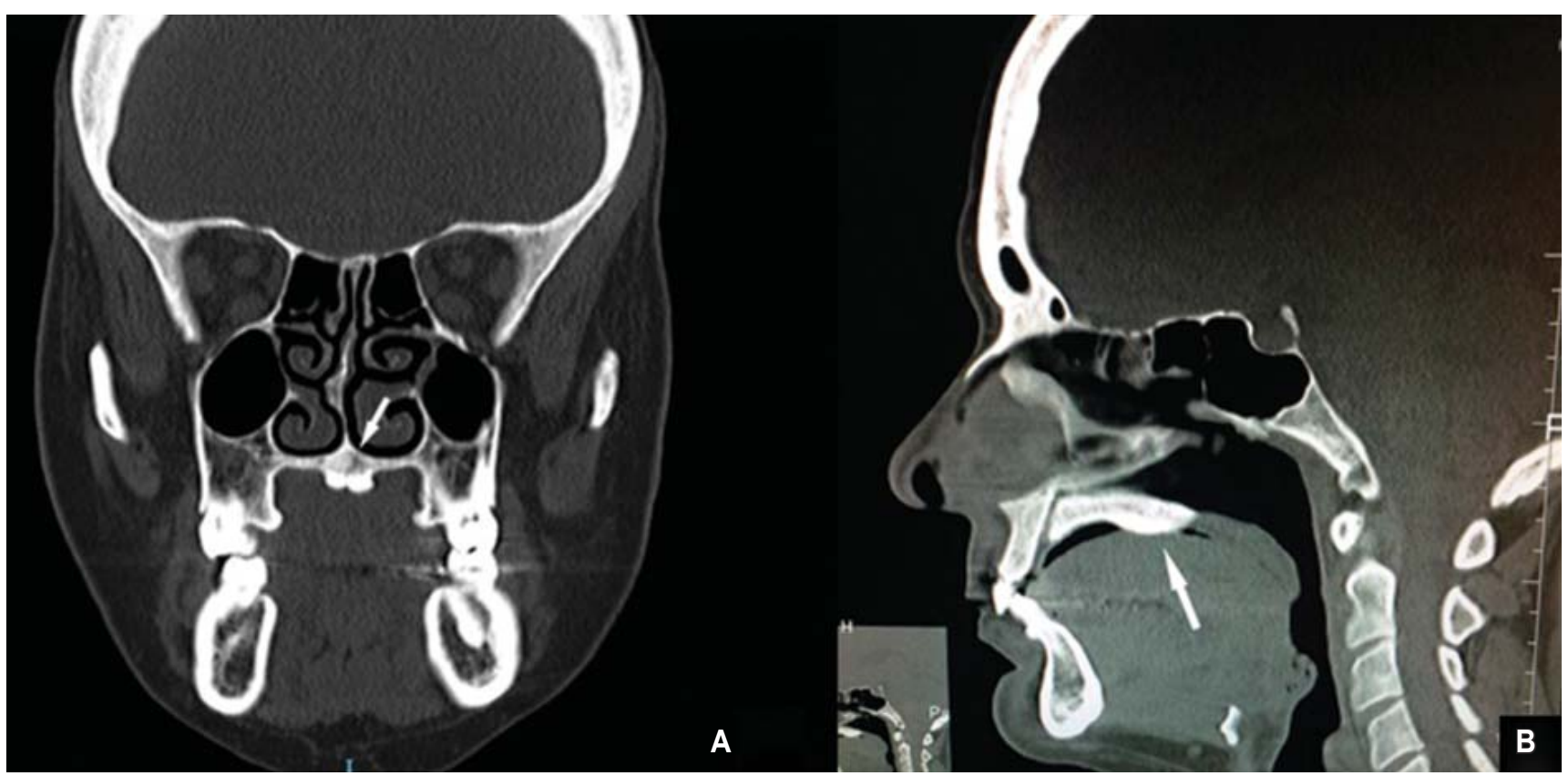

Figura 2: A) TAC corte coronal. B) Corte sagital donde se evidencia excrecencia ósea en la línea media palatina. 
Dentro de las complicaciones debemos recordar la exposición espontánea del torus con su posterior secuestro óseo. Esto se atribuye a múltiples causas: daño traumático por alimentos duros, cepillado, constricción vascular, aumento de fuerzas de oclusión o trauma quirúrgico. ${ }^{4,10}$ También se ha observado osteonecrosis asociada al uso de bifosfonatos..$^{5,10}$

Ocasionalmente el TP requiere tratamiento quirúrgico. ${ }^{1,7}$ La extirpación se realiza si obstaculiza la colocación de prótesis removibles, si el gran tamaño dificulta la deglución, masticación y fonación, cuando se requieren como fuente para injerto de hueso autólogo ${ }^{1,7,8,10}$ y cuando se producen úlceras que no cicatrizan y que podrían evolucionar a osteomielitis crónica ${ }^{8,9}$ o contribuir a las formaciones de lesiones malignas de la cavidad oral. ${ }^{8}$ En nuestra paciente sólo se tomó conducta preventiva y educación sobre su patología.

\section{CONCLUSIONES}

El torus palatino es una de las formaciones óseas benignas más frecuentes de la cavidad oral, la cual es importante considerar debido a los diagnósticos diferenciales a tener en cuenta, y recordar que el examen de la mucosa oral es parte del examen dermatológico, y que en esta se pueden encontrar patologías propias y también manifestaciones de enfermedades sistémicas.

\section{BIBLIOGRAFÍA}

1. Nogueira SA, Gonçales SE, Santos PS et al. Clinical, tomographic aspects and relevance of torus palatinus: case report of two sisters. Surg Radiol Anat. 2013; 35: 867-871.

2. Scrieciu M, Mercu V, Mercu R, Bîrjovanu C, Stan MC, Marinescu IR et al. Morphological and clinical characteristics of the torus palatinus and torus mandibularis in a sample of young and adults' Romanian people. Rom J Morphol Embryol. 2016; 57 (1): 139-144.

3. Batista ST, Figueredo EJ, Estrada MAR, Jardines GT, Tamayo AJ. Torus mandibulares y palatinos en pacientes atendidos en la Policlínica Pedro Díaz Coello. CCM. 2013; 17 (3): 315-319.

4. Sinisterra G, Álvarez J, Molano PE. Exposición espontánea de un torus palatino de la línea media. Biomédica. 2013; 33: 31-35.

5. El Achkar VN, Lopes SL, Pinto AS, do Prado RF, Kaminagakura E. Imaging aspects of palatal torus in cone beam computed tomography and magnetic resonance: case report. Acta Stomatol Croat. 2016; 50 (4): 359-364.

6. Lai WS, Shih CP. Triple torus palatinus. Indian J Med Res. 2014; 139 (5): 788.

7. Fuentes F, Borie EE, Sanhueza CA, Rebolledo SK, Parra VP. Presencia de exostosis orales en pacientes de la ciudad de Temuco, Chile. Av Odontoestomatol. 2012; 28 (2): 63-69.

8. Manotas Al, Estevez AE. Torus palatino, torus mandibulares y exóstosis de los maxilares en pacientes de la Cínica Odontológica de la Universidad del Magdalena. Año 2006. Rev Cienc Biomed. 2010; 1 (1): 47-53.

9. Marchena RL, Osorio RM, Sánchez FM. Motivo de tratamiento quirúrgico de un Torus en la consulta de Odontología. RODOE. 08/05/2014 12:00:01.

10. Ceccotti E. Exostosis benignas de los maxilares. Disponible en: www.intraMed.net. 